\title{
Neue Medien - neue Menschen?
}

\author{
von Manfred Becker-Huberti
}

\author{
Wenn Herr K. einen Menschen liebte: \\ „Was tun Sie“, wurde Herr K. gefragt, \\ „wenn Sie einen Menschen lieben?" - \\ „Ich mache einen Entwurf von ihm“, sagte Herr K., \\ „und sorge, daß er ihm ähnlich wird.“ \\ "Wer? Der Entwurf?" \\ - „Nein“, sagte Herr K., „der Mensch“.
}

Bert Brecht ${ }^{0}$

Zwei Reizthemen sind es, die einer anbrechenden Informationsgesellschaft auf den Nägeln brennen. Einmal ist es die - spätestens seit Günther Anders als infantil und antiquiert diffamierte ${ }^{1}$ philosophisch-anthropologische Frage nach dem Menschen. Zum Zweiten geht es um die zur ideologischen Standortbestimmung unvermeidlichen Frage nach unserer Haltung angesichts der prosperierenden Informations- und Kommunikationstechniken (=IuK-Techniken). Eine Korrelation beider kann deshalb nicht ohne Reiz sein. Eine Darstellung der in der Themenstellung intendierten Wechselbeziehung zweier so komplexer, weil ideologiebefrachteter Themen kann unter Intellektuellen, die weder Informatiker, höhere Postbeamte oder EDV-Fachleute sind, kaum Einseitigkeiten vermeiden.

In Anbetracht dieser Ausgangsposition seien eingangs einige Definitionen des Autors in der Absicht eines kurzen Credos vorausgeschickt:

a. Mir scheint die Frage nach dem Menschen, nach seinem Selbstverständnis, seinem Wesen und seiner Natur notwendiger denn je. Diese Frage läßt sich auch durch die Bemerkung von Anders nicht aus der Welt schaffen, daß es mit gleichem Recht dann wohl auch eine „philosophische Hippologie" geben könne ${ }^{2}$. Erst wenn man die Sonderstellung des Menschen im Kosmos und damit seine Geschöpflichkeit aufgibt und ihn als eine überholbare evolutionäre Entwicklung ${ }^{3}$ betrachtet, wird es für den Menschen unerlaubt, die Frage nach sich selbst zu stellen. - Dies aber auch nur, weil sie sich nicht mehr beantworten läßt!

b. Die Vorstellung, alle Wirklichkeit lasse sich sauber in „Gut" und „Böse“ teilen, halte ich für präpubertär. Heutzutage werden mit schmerzlicher Aufdringlichkeit Lösungen aller Probleme in „guten“ oder „bösen“ Varianten angeboten. Ich halte diese für plump und dümmlich. Die Verkünder bipolarer Lösungen verkennen die schlichte Einsicht, daß sich Moral meist durch den Gebrauch der Dinge ergibt, nicht so sehr durch die Dinge selber.

Dr. theol. Manfred Becker-Huberti ist katholischer Theologe und Publizist. Er leitet die Katholische Bildschirmtext-Redaktion Düsseldorf, eine Einrichtung der Bistümer in Nordrhein-Westfalen. Der Autor gehört zur Redaktion von Communicatio Socialis. 
c. Die Vorstellung, es gebe ein sinnvoll anzustrebendes Ziel, das sich mir ohne mein Dazutun auftue, dessen Erreichen sich ebenso traumhaft vollziehe, ist unrealistisch. Der Mensch muß definieren, welches Ziel er hat und auf welchem Weg er dieses Ziel zu erreichen versucht!

Weil in den modernen Informations- und Kommunikationstechniken ebensowenig "Sinn“ enthalten ist wie er etwa im Buch enthalten war, muß dieser „Sinn“ definiert werden. Zielgerichtete (teleologische) Bestimmungen setzen aber voraus, daß derjenige, der Sinn und Ziel definiert, über eine klare, unmißverständliche Ausgangsposition verfügt: sein Selbstverständnis, seine Werte und Normen müssen evident sein.

Eben dies ist gegenwärtig gesamtgesellschaftlich weder zu leisten, noch sind sich weite Kreise der Gesellschaft darüber im klaren, daß dieses Problem besteht. Dies ist um so verwunderlicher, als eben diese Gesellschaft darunter leidet, daß alte Zielvorgaben fraglich geworden sind: Fortschritt, Einsatz der Technik für ein leichtes, glückliches Leben, Wohlstand, Sicherheit ...

Lösungsansätze lassen sich wohl am ehesten finden, wenn diese Gesellschaft wieder ein möglichst homogenes Selbstverständnis vorweisen kann, wenn also einzelne gesellschaftliche Gruppen ihre Vorstellungen vortragen und abstimmen.

Nachfolgend soll im vorgenannten Sinn der Versuch einer anthropologischen Ortsbestimmung und teleologischen Ausrichtung versucht werden.

\section{Vom homo sapiens zum homo informatus}

„Quid est homo?“, so lautet seit alters die Frage des Menschen nach dem, wer und was er sei. Die auf diese Frage gegebenen Antworten sind Legion ${ }^{4}$. Anthropologien im positiven Sinn (und manchmal auch im negativen) gibt es in größerer Zahl; fast jeder Philosoph, der auf sich hielt, versuchte sich auch auf diesem Gebiet ${ }^{5}$. Keiner hat aber so eindeutig klar formuliert wie Kant: „Das Feld der Philosophie . . läßt sich auf folgende Fragen bringen:

1 Was kann ich wissen?

2 Was soll ich tun?

3 Was darf ich hoffen?

4 Was ist der Mensch?“

Kant ordnet dann für seine Zeit treffend zu: „Die erste Frage beantwortet die Metaphysik, die zweite die Moral, die dritte die Religion, und die vierte die Anthropologie". In unserem Zusammenhang besonders interessant ist der nachfolgende Satz Kants: „Im Grunde könnte man aber alles dieses zur Anthropologie rechnen, weil sich die drei ersten Fragen auf die letzte beziehen." ${ }^{\text {“6 }}$

In allem, was durch Menschen hervorgebracht wurde und wird, kommt das Menschsein selbst zum Ausdruck: Wissenschaft und Religion, Kunst und Literatur, Rechtsordnung und Staatsverfassungen liegt ein bestimmtes menschliches Selbstverständnis zugrunde. Dieses menschliche Selbstverständnis ist oft nicht ausdrücklich als Anthropologie ausformuliert, meist nicht einmal reflektiert, sondern wird als Selbstverständlichkeit 
vorausgesetzt und/oder durch sprachliche Begrifflichkeiten und Formulierungen ausgedrückt. Die Bezeichnung des Menschen als „Geschöpf Gottes“ oder als „informationsverarbeitendes System mit input und output" sind Deutungen, denen religiöse oder informatorisch-kybernetische Modelle zugrundeliegen. Auf diese „implizite Anthropologie“ oder „Kryptoanthropologie“, „die menschliches Selbstverständis durch sprachliche Formulierung zum Ausdruck bringt, die sich aus Übertragungen aus anderen Wissenschaften ergibt“, hat Rudolf Lassahn hingewiesen?.

Lassahns Feststellungen, da $\beta$ in den verschiedenen Problemstellungen, in denen die Anthropologie nicht einmal thematisiert ist, das Menschenbild zur Diskussion steht (etwa in der Gesellschaftslehre, Erkenntnislehre oder physiologischer Basis der Erkenntnis), entspricht die parallele Feststellung, daß das Menschenbild die Muster und Begriff-lichkeiten zu seiner eigenen Beschreibung jeweils dem Erkenntnisstand der zeitgenössischen Wissenschaft entlehnte; spätestens seit dem Beginn der Neuzeit lieferten so die Naturwissenschaften die Vorlagen zur Formung der Lehre vom Menschen.

„Die Maschinen, die sich der Mensch konstruierte, dienen nicht nur der Arbeitserleichterung. Sie sind auch Abbild und Spiegelung der menschlichen Weltsicht. Die astronomische Weltuhr zur Zeit der Dynamik repräsentierte die beiden Aspekte: sie bildete das Weltall mit den Planetenbewegungen ab, sie war aber auch Abbildung der Auffassung, Welt und Mensch als kunstreiches Uhrwerk zu sehen. Man suchte die Gesetzmäßigkeit im Weltall, aber auch im Menschen. Das Welt- und Menschenbild determinierte das Handeln. Ursprünglich hatte die Uhr die Zeit zu messen. Sie war Instrument. Aus der Meßbarkeit der Zeit folgte im menschlichen Leben deren Regelung. Alle täglichen Verrichtungen, die Arbeitsabläufe in der Industrie, aber auch unser Tagesablauf werden jetzt durch die Uhr geregelt. Die Uhr ist keine Prothesenmaschine, sie verstärkt nicht irgendeinen Funktionskreis menschlichen Tuns. Die Uhr dient jetzt der Rationalisierung der Zeit in mechanischer Vereinseitigung und trennt damit von der Lebenswelt. Wir kennen nicht mehr den ,Tageslauf, eine Vorstellung abgeleitet vom Lauf der Sonne und der Gestirne, sondern die, Tageszeit'. Wir essen nicht, wenn wir Hunger haben, sondern wenn ,Mittagszeit' oder ,Essenszeit' ist; wir stehen nicht auf, wenn wir ausgeschlafen sind, sondern wenn der Wecker klingelt.“8

Für die Zeit der Dynamik war die Uhr das große Vorbild, von ihr wurde die mechanische Kraftübertragung in der Maschine abgeleitet. Ihr folgte die Wärmemaschine, die immer weiter verbessert wurde und zugleich zum Modell für menschliches und gesellschaftliches Leben wurde. Die Antriebskraft der Wärmemaschine waren nicht mehr menschliche oder tierische Kraft oder die Elemente Wasser oder Wind. An ihre Stelle trat die Energie; die Energie wurde durch Arbeit in Leistung umgesetzt. In der Epoche der Thermodynamik wurde ein berechenbarer Zusammenhang zwischen Energieaufwand und Leistung hergestellt. ${ }^{9}$

Die Arbeitskraft einer Maschine war berechenbar. Dies zog nach sich, daß auch die Arbeit des Menschen berechnet wurde.

Noch schlimmer: die Bedeutung des Menschseins konnte in der Berechnung der Arbeitskraft aufgehen: Karl Marx reduzierte den Lohnarbeiter konsequent auf seine Arbeitskraft. Die Grundbegriffe der Thermodynamik - Energie, Arbeit und Leistung prägen von diesem Zeitpunkt an das philosophische und pädagogische Denken (Comenius, Herbart). Die mathematische Fassung der Relation von Energie, Arbeit und Leistung führte zur Optimierung des Funktionsablaufes (Minimax-Theorem): jeder Faktor im System hat seine bestimmte Funktion, deren Wert durch andere Systemeigen- 
schaften bestimmt wird. Die Berechenbarkeit der Leistung verstärkt die Bedeutung des Preises. Kosten gestalten sich in Relation zum Nutzen der geleisteten Arbeit. Die unkritische Adaption thermodynamischer Termini in die implizierte Anthropologie greifen bis heute. ${ }^{10}$

Die Homologisierung" von Worten aus dem neu entdeckten Elektromagnetismus in die implizite Anthropologie sind vergleichsweise harmlos. Gleichwohl scheinen diese Begriffe in einer kaum noch vorstellbaren Weise fasziniert zu haben. „Pol“ und „Polarität", „Spannung“" und „Spannungsverhältnis“, „Widerstand“, „Kontakt“ und „Netzwerk“ werden nicht nur zu umgangssprachlichen Termini, die das Selbstverständnis ausdeuten. Sie werden auch zu Topoi von Wissenschaften, die bis dahin den Naturwissenschaften die Termini vorgegeben hatten. Die Naturphilosophie Schellings verwendet die Worte „Pol“ und „Polarität". „These“ und „Antithese“ werden zu polaren Ausdrücken. Das Spannungsgefüge eines Charakters (Nohl) führt bis in die Gegenwart zu Wortbildungen wie „Kontaktperson“, „Sozialkontakt“, „Kontaktzentrum“ und ähnlichem.

Entscheidend für uns heute wurden jedoch die Homologisierungen aus dem Bereich der Kybernetik und Informatik: „Die Konstrukteure des dynamischen Systems entdeckten die Kraft und fanden eine Formel zu ihrer Berechnung; die Thermodynamiker verwandelten Kraft in Energie, und auch sie fanden Wege zur Berechnung bis hin zum Minimax-Theorem. Die Kybernetiker schließlich verwandelten Energie zur Information" ${ }^{12}$. Information ist Information, nicht Materie und nicht Energie, so definierte Norbert Wiener ${ }^{13}$ tautologisch. Steinbuch wies dem Begriff Information die gleiche Bedeutung zu, die Kraft und Materie in vergangenen Zeiten hatten, - und es sieht so aus, als ob sich seine Ansicht bewahrheite.

Information wurde zu einer dritten Kategorie. Bei der Information handelt es sich nicht um Materie oder Energie, sondern um eine Struktur. Information wurde definiert als eine vom „sinnlichen“ Gehalt abstrahierte Struktur, Kybernetik als mathematische Strukturtheorie (Wiener, Shannon, Weaver) ${ }^{14}$. „Die Verbindung der Kybernetik mit der Systemtheorie (Bertalanffy, Ashby, Hall und Fagan; in Deutschland Lohmann), Spieltheorie (J. von Neumann und O. Morgenstern; in Deutschland Eigen und Winkler) und der behavioristischen Psychologie (Skinner und die Neubelebung der Reflexionstheorie von Pawlow) erbrachte jene Muster, die innerhalb von vierzig Jahren alle Wissenschaften erfaßten: Information, Kommunikation, System, Steuerung, Regelung und Verhalten." 15

War in der Dynamik alles Kraft, in der Thermodynamik alles Energie, so ist jetzt alles Information; Steinbuch listet einfach: Gesang eines Vogels, Grafik von Picasso, Briefe jeden Inhalts, Fotografie und Film, Literatur vom Telefonbuch bis zur Bibel, Gesetze und Verordnungen, Musik vom Trommelschlag bis zur neunten Symphonie, die Gene, welche die Erbeigenschaften übertragen und andere ${ }^{16}$.

„Der Mensch empfängt Informationen, er sammelt und speichert sie, entweder im Kurzzeit- oder Langzeitspeicher, Informationen werden verarbeitet" weitergegeben, in der Gesellschaft gibt es einen ständigen Informationsfluß, es gibt gut und schlecht informierte Kreise, der Informationsfluß stockt oder wird unterbrochen, Informationen werden zurückgehalten, entstellt oder verfälscht. Alles ist Information. Die Gene übermitteln Informationen, die Zeitungen drucken sie, Informationen steuern den Verkehrsfluß, die Kühlschränke und elektrischen Schreibmaschinen, regeln die Waschmaschinen und den Produktionsverlauf in einer Autofabrik. 
Innerhalb dieser ,großen, gewaltigen geregelten und gesteuerten Systeme ist der Mensch nur ein kleines Subsystem unter vielen anderen. Er ist ein der Selbstregulation befähigtes System ... Der Mensch wird dabei aber auch ein System, das man - beliebig wünschen viele - steuern und regeln kann“17.

Die anthropologische Dimension hinter dieser Ausprägung der Kybernetik gipfelt in der Erkenntnis von G. Günther: „Da man aber nur wirklich das versteht, was man macht ..., bleibt dem Menschen jetzt nichts anderes übrig, als einen Versuch zur Selbstinterpretation dadurch zu unternehmen, daß er sich in einer technischen Nachbildung wiederholt. Diesem Z weck dienen alle kybernetischen Formeln, Theorien und Mechanismen “18. In der Folge dieser Erkenntnis waren die Versuche zur Selbstinterpretation des Menschen durch das Muster von Apparaten nur konsequent. ${ }^{19}$

Fügen wird zusammen: die Kryptoanthropologie vermag aufzuzeigen, in welchem Ausmaß der Mensch sein Selbstverständnis aus dem jeweiligen Erkenntnisstand der Naturwissenschaften bezieht. Diese implizite Anthropologie definiert den Menschen durch Vorgaben, Muster und Klischees der Naturwissenschaften - einer Wissenschaft, die sich der Natur nicht mehr nur angenommen, sondern sie abgebildet und auch verändert hat. Sie droht nun den Menschen zum Gegenstand eines Veränderungsprozesses zu machen.$^{20}$ Die Neuschaffung des Menschen, einzeln schon die Auflösung der Menschheit durch „intelligente“ Maschinen, steht an. ${ }^{21}$

An diesem Punkt ist das ursprüngliche Ziel menschlichen Seins angefragt. Es ist eine Anthropologie, die den autonom handelnden, selbstkritischen und humanen Menschen, das Ich-Selbst, zum Ziel proklamiert. Diese Ziele gelten aber nicht mehr, wenn sich der Mensch selbst zum Gegenstand eines Veränderungsprozesses oder durch eine Neu_,Schöpfung“ sich selbst überflüssig macht.

Nicht nur das Ziel der herkömmlichen Anthropologie droht aufgegeben zu werden, auch die angewandte Methode kann nicht zum Ziel führen: wer sein Selbstverständnis vom selbstgeschaffenen, reduzierten Abbild gewinnen will, wer sich selbst als determiniert, außensteuerbar entwirft, kann nicht emanzipierte Menschen erziehen.

Wenn der Mensch so handelt wie er sich sieht, wenn sich der Mensch seine Vorbilder selbst entwerfen muß, dann bleibt die Übernahme von der Anthropologie fremden Methoden, Verfahren und Denkweisen nicht ohne Rückwirkung. Die Ansicht, alles skalieren, alles in mathematische Formeln fassen zu können, alles auf systemgültige Formeln und Gesetzmäßigkeiten zurückführen zu können, alles steuer- und regelbar machen zu können, führt in der Anthropologie unweigerlich zu einer Teilansicht des Menschen. Der Mensch wird Krüppel, Android, homunculus. Von diesem Abbild bezöge der Mensch sein Selbstbild.

An dieser Stelle müßte begonnen werden (hätte längst begonnen werden müssen!), das Grund-Selbstverständnis des modernen Menschen neu und verständlich zu definieren. Dies muß auch geschehen auf die Gefahr hin, daß es zu keiner gesamtgesellschaftlichen Antwort, sondern zu alternativen Antworten kommt. Besonders die Kirchen haben hier ihre Aufgaben.

Statt Antworten gibt es heute zur vorgenannten Thematik viele Fragen und Ängste. Die Zukunft ist mancherorts doch längst Gegenwart ${ }^{22}$; zahlreiche Menschen fragen sich, ob sie nicht in einem ihnen unbekannten Fahrzeug zu einem unbekannten Ziel unterwegs sind. Zielsetzung, Planung und Bedingung der Informationsgesellschaft scheinen undurchsichtig und ängstigend. Die Reduktion der Wirklichkeit auf bloß empirische 
Wirklichkeit und/oder medialvermittelbare Wirklichkeit ahnen und empfinden sicherlich mehr Menschen, als sie selbst mitzuteilen in der Lage sind. Dies geschieht zu einem Zeitpunkt, an dem feststeht, daß nicht nur verpaßt worden ist, die Gesellschaft auf diesen Wandel vorzubereiten; viel wichtiger ist, daß wohl auch vergessen worden ist zu diskutieren, welche Ziele $\mathrm{zu}$ welchem Nutzen und $\mathrm{zu}$ welchen Bedingungen erreicht werden sollen!

\section{Die Informations- und Kommunikationstechniken ändern unsere Welt}

Indikatoren der gegenwärtig laufenden Verbreitung der IuK-Techniken sind vielen Menschen der Stand der Verkabelung, der Siegeszug des Videorecorders, der Einzug des Computers in Büros und Schulen. Dabei wird leicht übersehen, was der laufenden Entwicklung an Zielsetzung vorausgeht und welche Bedingungen sie eigentlich ermöglichen.

Technischer Zielpunkt der fortschreitenden Ausbreitung der IuK-Techniken ist die Verwirklichung des „Kommunikationskabels“, über das jede Form von Kommunikation von jedem zu jedem in beliebiger Menge, zu beliebiger Zeit ablaufen kann.

Dieses Kabel wird als ein Breitbandkabel um den Jahrhundertwechsel in der Bundesrepublik verwirklicht sein. Es wird die Kommunikation in Form von Sprache, Text, Bild und Daten zeitgleich erlauben und uns in Wohnung oder Haus eine „Kommunikationssteckdose" bescheren, die Schnittstelle für ein anschließbares multifunktionales Gerät oder mehrere Einzelgeräte sein kann. Mit Hilfe dieser Geräte oder des einen Gerätes lassen sich Texte dokumentieren und archivieren, Daten verfassen und verarbeiten, Bilder erzeugen und verarbeiten. Das Kommunikationskabel wird Informationen in immaterialisierter Form digitalisiert mit Lichtgeschwindigkeit verbreiten: Text, Bild, Sprache und Daten werden codiert in binäre Ziffern und nicht mehr elektrisch und analog, sondern mit Lichtgeschwindigkeit und digital übertragen.

Die Bausteine und Basisinnovationen dieser Möglichkeiten sind die Mikroprozessoren, die Möglichkeit der Digitalisierung und die Entwicklung der Lasertechnik. Mit Hilfe der Mikroprozessoren, miniaturisierten hochkomplexen Schaltungen, lassen sich Rechenoperationen und Steuerungsprozesse durchführen. Diese Bausteine haben den Vorteil, $\mathrm{da} ß$ sie immer kleiner werden, immer höhere Leistungen erbringen und dennoch immer preiswerter werden. ${ }^{23}$ Die Digitalisierung führt zur beliebigen Kopierbarkeit von erfaßbaren Informationen der unterschiedlichen Kommunikationsarten unter der Bedingung, daß immer eine 100prozentig einwandfreie Kopie geliefert wird. In Verbindung mit Mikroprozessoren und Lasertechnik eröffnet die Digitalisierung ungeahnte Quantitäten der Speicherung, Übertragung und Verarbeitung von Information. Die Lasertechnik ermöglicht die Informationsübertragung mit Lichtgeschwindigkeit in allen Frequenzbereichen. Sie steigert die Übertragungsleistung gegenüber der alten Technik mindestens um den Faktor 1.000 und befreit von der Sorge um das Kupfer, das als Grundlage jetziger Technik immer seltener und darum immer teurer wird.

Diese IuK-Techniken bedeuten nicht einfach nur ein mehr an Kommunikation: sie ermöglichen die Quantifizierung von Zeit, von Raum und von Werten (Geld). ${ }^{24}$ Sie heben die bisherige Unterscheidung von Massen- und Individualkommunikation auf. Sie verändern den Medienmarkt und haben das Medienrecht bereits aus den Angeln gehoben. Auch weitere Rechtsgrundsätze werden die IuK-Techniken in Frage stellen, ${ }^{25}$ und sie sind 
Indiz für ein sich wandelndes Wirtschaftssystem, in dem die Information mit einem Anteil am gesamten Markt von über 50 Prozent zur Ware wird. Über die Quantifizierung von Raum, Zeit und Werten hinaus führen die IuK-Techniken in eine neue Dimension von Kommunikation: ontische (seinsmäßige) Veränderungen von diesem Ausmaß bleiben ontologisch (Wesensbestimmung des Seienden) nicht folgenlos. Die massenhafte Verfügbarkeit von Unterhaltungsangeboten und Informationen ermöglicht medialen Konsum in ungekannten Mengen und Formen von Datenverarbeitung in noch nicht bewußten Anwendungsbereichen. Kommunikationsaskese wird wahrscheinlich nur partiell möglich sein, weil Bedingungen geschaffen werden, die den vollkommenen Verzicht unmöglich machen: das elektronische Telefonbuch, der elektronische Kontakt mit der Bank, die elektronische Post im Datenspeicher et cetera. Die totale Dezentralisierung wird verwirklicht bei gleichzeitig vollständiger Zentralisierung der Macht.

Der Mensch steht einer IuK-Technik gegenüber, die ihn weiterhin langsam, ungenau und psycho-physisch anfällig sein läßt; er vermag weiterhin immer nur wenige Zusammenhänge zu überblicken, ist an die engen Grenzen von Raum und Zeit gebunden: vergeßlich und kritisch, aber kreativ und intuitiv. Demgegenüber ist der Rechner milliardenfach schneller, arbeitet mit inhumaner Präzision, Zuverlässigkeit und Ausdauer, überwindet Raum und Zeit und ist unvergeßlich. ${ }^{26}$

Wird die Menschheit ihr Bild von sich durch die technischen Möglichkeiten der IuKTechniken bestimmen lassen oder werden die Menschen ihre spezifischen Fähigkeiten und die der Rechner und Übertragungsnetze kennen- und unterscheiden lernen? Wird die Wirkung der IuK-Techniken auf den Menschen vielleicht doch noch ganz anders sein und ihn in einer Weise determinieren, wie Günther Anders befürchtete? Anders kleidet seine Angst in eine Geschichte: „Da es dem König aber wenig gefiel, daß sein Sohn, die kontrollierenden Straßen verlassend, sich querfeldein umhertrieb, um sich selbst ein Urteil über die Welt zu bilden, schenkte er ihm Wagen und Pferd. „Nun brauchst du nicht mehr zu Fuß zu gehen“, waren seine Worte. „Nun darfst du es nicht mehr“, waren deren Sinn. „Nun kannst du es nicht mehr“, deren Wirkung. ${ }^{27}$

Die Unsicherheiten und Ängste der Menschen drücken sich - nicht nur in der Literatur! - in zahlreichen Formulierungen aus. Hier eine (natürlich unvollständige) Liste von befürchteten Auswirkungen der IuK-Techniken:

- die Gefahr eines Computer- und Überwachungsstaates

- der Verlust von Arbeitsplätzen

- die Flucht in eine Medienscheinwelt

- die zunehmende Ungleichheit der Bildungschancen

- die neue Korrelation von Mensch und Maschine

- die Verdrängung der zwischenmenschlichen Kommunikation

- die Durchsetzung des Maschinendenkens als vorherrschendem Denkmodus

- die Verwundbarkeit einer von technischen Großsystemen abhängigen Gesellschaft

- die verschärfte Geschlechtsrollenzuweisung

- die Reduktion der Schriftsprache auf Textverarbeitung

- die Dominanz der Bilder

- die Anonymisierung von Wissensvermittlung

- die Aufweichung des öffentlichen Bildungsauftrages.

Das technische Ziel der Informationsgesellschaft, das aus Rationalisierungsgründen verfolgt wird, wird - wenigstens vorübergehend - Arbeitslose produzieren. Nicht nur die Frage nach dem Sinn von Arbeit muß neu beantwortet werden, es müssen auch 
humane Lösungen gefunden werden für die, die den Anforderungen der neuen Entwicklungen (noch?) nicht gewachsen sind: die Arbeitslosen, die infolge der technischen Entwicklung existieren, aber auch die Behinderten, ältere Menschen, die „Unqualifizierbaren“ (Haefner). Wer tritt heute dafür ein, daß die Humanität der Informationsgesellschaft morgen daran zu messen ist, wie diese Gesellschaft mit denen umgeht, die sie nicht problemlos integrieren kann?

\section{Die Bildung am Ende des Gutenberg-Zeitalters}

Die ontischen Veränderungen legen die Empfehlung nahe, daß die Instanzen, die ausfort- und weiterbilden, sich auf die neuen Bedingungen einstellen. Auf das Gesamt gesehen, scheint diese Annahme noch irrig und wohl verfrüht. Neben der Tatsache, daß in den meisten weiterbildenden Schulen unterschiedliche Computer und unterschiedliche Software eingeführt wurden - und dies meist nicht durch die Schulträger oder Kultusminister, sondern durch engagierte Lehrer und Eltern - werden in den gymnasialen Oberstufen Informatik-Kurse angeboten ${ }^{28}$, finden an Kaufmännischen-Berufsbildenden Schulen entsprechende Kurse statt. Grundlegende Einführungen in Telekommunikation oder Telematik, ihre Bedingungen und Arbeitsweisen, Anwendungsmöglichkeiten und gestaltende Prinzipien, wirtschaftliche Faktoren und ähnliches, finden so gut wie gar nicht statt. Und das nimmt auch nicht Wunder. Wer sollte sie durchführen, mit welcher Technik, unter welchen curricularen Vorgaben?

Außer einer diffusen Angst vor dem Neuen ist in vielen Schulen noch nicht viel angekommen, geschweige in den meisten Lehrerseminaren. Ganz allmählich scheint sich an einzelnen Hochschulen außerhalb der Naturwissenschaften die „Informationswissenschaft" als Begleitstudium zu etablieren, scheinen Geisteswissenschaftler neue Aufgabenfelder zu entdecken. Die wenigen Vorreiter, die es hier gibt, kämpfen gegenwärtig meist noch gegen eine uneinsichtige Kultus- und Hochschulbürokratie. In den Bereichen der Fort- und Weiterbildung ist die zweckorientierte, bedarfsgerechte Ausbildung von EDV-Leuten geordnet. Die fach- und berufsübergreifende Sicht der Probleme also die Fragen nach Struktur und Sinn, Entwicklungstendenz und Ziel, werden hierbei nicht oft dargestellt. Außerhalb der Ausbildungskurse laufen Kurse für Hobbyisten und Interessierte. Über diese schmalen Anwendungsbereiche hinaus gibt es nur wenige Angebote, die alle das Problem haben: was soll eigentlich wie, wem mit welchem Ziel erläutert werden? Angesichts dieses Konflikts und dem meist fehlenden Problembewußtsein der Bevölkerung unterbleibt ein Engagement der Erwachsenenbildner. Für sie gilt überdies (das behaupte ich aus Erfahrung und nicht aufgrund von Befragungsergebnissen), daß sie, die methodisch fast ausschließlich auf face-to-face-Kommunikation ausgerichtet sind, mit Unbehagen und Mißtrauen Formen technischer Kommunikation begegnen. Tendenziell möchten Erwachsenenbildner ihre tradierten Formen beibehalten.

Diesem aus der Sicht des Autors wenig erfreulichen Gesamtzustand des Bildungsangebotes steht die Notwendigkeit gegenüber, auf die Informationsgesellschaft vorbereitet $\mathrm{zu}$ werden. Es genügt nicht, auf die verschiedenen Formen der Anwendung der Telekommunikation hingewiesen zu werden, sie muß geübt und erprobt, Schwächen und Stärken erfahren werden. Das Kennenlernen darf sich nicht auf die Anwendung beschränken, sondern muß die Strukturen der Technik, die Organisation der Bedingungen et cetera aufzeigen. Es muß vor allem endlich Ziel der Erziehung werden, instru- 
mentelles und topisches Denken ${ }^{29}$ unterscheiden und anwenden zu lernen. Wo eigentlich sollen die Veränderungen geistig bewältigt werden, wenn nicht in der Schule, Hochschule oder in Einrichtungen der Erwachsenenbildung? Und ein Letztes hierzu: wenn infolge der IuK-Techniken die Inhalte einzelner Wissensbereiche für die neuen IuK-Techniken aufzubereiten sind, wird sich die Frage stellen, wer dies tun soll: die Wirtschaft nach ökonomischen Gesichtspunkten oder die Pädagogen nach ihren Zielvorstellungen (welchen?)? Wenn die Wirtschaft nach ihren Gesetzmäßigkeiten in diesen „Markt“ eindringen wird, wie erste Anzeichen andeuten ${ }^{30}$, dann definiert sie die Methoden und Ziele und läßt unbearbeitet, was keinen Profit verspricht. Die Verhinderung profitorientierter Bildung war aber mit ein Grund zur Errichtung des staatlichen Bildungsmonopols. Solange aber die Finanzminister die Bedingungen der Kultur definieren und solange die Pädagogen nicht ihre eigentliche Aufgabe, die qualifizierte Vorbereitung einer neuen Generation auf die Zukunft, bei ihrer eigenen Vorbereitung beginnen, ist aus den Schulen wenig zu erwarten.

\section{Ist Information eigentlich Selbstzweck?}

Die Definition des Menschen in der Informationsgesellschaft als ein „sapiens et informatus" geht von der Prämisse aus, daß dieser Mensch ein „sapiens“ sein muß, um ein „informatus" sein zu können. Dies ist eine gewagte Behauptung, doch unausweichlich notwendig: denn wenn Information nicht Selbstzweck sein soll, die Quantität der Information nicht allein schon Legitimation ist, dann muß der „homo sapiens“ Kriterien für die Selektion und den Gebrauch von Informationen entwickeln. Hinter den Anfragen an Technik, Fortschritt und Wissenschaft stehen die ethischen und anthropologischen Fragen. Die Idee, man müsse nur der menschlichen Vernunft und den von ihr angewandten exakten Methoden vertrauen, dann werde die Menschheit in eine neue, nie gekannt euphorische Menschheitsphase eintauchen, kann heute niemand mehr guten Wissens verbreiten. Die Rede von ,den exakten Wissenschaften"selbst wird in Zweifel gezogen. Der Anspruch auf die Gültigkeit naturwissenschaftlicher Experimente ist längst reduziert auf die durch das Experiment abgesteckten Bedingungen. Die erhoffte „absolute Wahrheit" ist so nicht zu gewinnen, nicht einmal eine „objektive Wahrheit" ist so $\mathrm{zu}$ erstellen. ${ }^{31}$

Eine zweite Erfahrung: Wenn Wissenschaft auf „Nützlichkeit“ und „Brauchbarkeit“ reduziert wird, verliert sie ihre Freiheit. Wenn sie sich von Bedürfnissen und Notwendigkeiten des Daseins in den Griff nehmen läßt, kann sie sich nicht mehr spontan der Wahrheit zuwenden. Gefährlich werden Wissenschaften dadurch, daß sie als exakte Wissenschaften einen totalitären Wissenanspruch erheben, einen Anspruch auf letztgültige Einsichten in Gesetze und Sinnwerte des Menschseins. Ein solches Selbstverständnis hat zwei offensichtliche Prämissen: a) der Mensch ist erkennbar und machbar und b) das wissenschaftlich und technisch Machbare ist auch das Gute. Die ethischen Implikationen von Wissenschaft und Technik werden zwangsläufig verkannt, weil alles Unantastbare und Nicht-Operable nicht akzeptiert werden kann.

Eine dritte Erfahrung: Karl Jaspers ${ }^{32}$ hat erklärt, daß die Menschheit durch die Erfindung und den Einsatz der Atombombe in eine neue Situation geraten sei: die Wissenschaft hat den Menschen in die Lage versetzt, sich selbst und andere radikal auszulöschen. Die These, daß das wissenschaftlich Machbare auch das Gute sei, sei durch diese Entwicklung falsifiziert, habe ihre Legitimation verloren. Alfons Auer folgert: „Das 
Sittliche, einst mit dem Rekurs auf Wertfreiheit aus der Wissenschaft verwiesen, meldet sich nunmehr auf dem Weg über deren Folgen wieder zurück. ${ }^{433}$

Angesichts dieser Feststellungen wird die Frage nach dem, was der Mensch denn nun tun soll, dringlich. Zahlreiche Fragen stehen heute an, auf die es bislang keine einheitlichen Antworten gibt, ja einvernehmliche Antworten zeichnen sich in naher Zukunft nicht einmal mehr als Möglichkeit ab.

Es sind die Fragen

- nach dem Selbstverständnis des Menschen überhaupt

- nach dem Sinn menschlicher Arbeit, ihrem Wesen und ihrer Rückwirkung auf den Menschen (Arbeit = Lohnarbeit = sinnspendendes Sein?)

- nach dem, was Technik und Fortschritt der Technik legitimiert (ist wissenschaftlich und technisch zu tun erlaubt, was sich verkaufen läßt?)

- nach der Korrelation von Natur - Mensch - Technik

- nach den Werten, die zu leben sich lohnen

- nach den Zielen, die anzustreben sich lohnen

- nach dem Sinn der Kommunikation zwischen Menschen und anderen/anderem

- nach dem Sinn von Information und Informationsverarbeitung

- nach der sozialen Rolle der „Nicht-Qualifizierbaren“

- nach den Triebfedern der laufenden Entwicklung

- nach dem Verbleib der tradierten Kulturtechniken

- nach den sich mit der laufenden Entwicklung verändernden Denk- und Verhaltensweisen

- nach den Faktoren, die als Meßschnur zur Bewertung und Zielsetzung herangezogen werden

- nach „Ort“ und „Wertsetzern“ einer Ethik der Technik

Angesichts dieser Problemstellung vertritt der Moraltheologe Alfons Auer die Ansicht, das Sittliche bezeichne den Anspruch der Wirklichkeit an die menschliche Person. Das Maß des Richtigen liege in jeder menschlichen Situation und in jeder Konstellation von Tatsachen und menschlichen Bestrebungen. Es heißt bei Auer weiter nachdrücklich, das Sittliche sei das Wirklichkeitsgemäße. Wahres Sein der Wirklichkeit, die innere Wahrheit der Dinge sei Maß und Norm des Handelns. Das Sittliche ist also der Wirklichkeit immanent, nicht transzendent. Eine „Ethik der Medien“ ist darum auch eine „immanente Ethik". Daraus folgt: im Prozeß der sozialen Kommunikation handelt sittlich richtig, wer sachgerecht handelt, wer die in diesem Bereich geltenden Gesetzlichkeiten und die vorgegebenen Sinnziele respektiert. Keinem Teilnehmer am sozialen Kommunikationsprozeß werden Normen von außen aufoktroyiert. Sittliche Normen oder Weisungen sind nur dann begründet, wenn die in ihnen artikulierten Verbindlichkeiten als innere Momente der sozialen Kommunikation erkennbar sind, im medialen Vermittlungsprozeß selbst ihre Dringlichkeit anmelden. Die innere Wahrheit der Dinge zu erkennen, ermöglicht die „Sachlichkeit“. Sie ist in der Lage, die wirkliche Gestalt eines Lebensbereiches, sein Zurückbleiben hinter den gegebenen Möglichkeiten und seine wesenhafte Hinordnung auf eine optimale menschliche Daseinsentfaltung wahrzunehmen. ${ }^{34}$

Bei aller Verschiedenheit ihrer Methoden zielen Wissenschaft und Technik in ihren gegenständlichen Bereichen immer auf den Menschen. Darum lassen sich Wissenschaft und Technik auch nicht von ethischer Einsicht und Sinngebung trennen. Ein Beispiel mag dies verdeutlichen: die Maximierung wirtschaftlichen Wachstums sollte den 
Lebensstandard des Volkes heben. Diese liberalistisch-kapitalistische Konzeption schien funktionsfähig, bis die Technokraten selbst erkannten, daß ein Kollaps des Systems in greifbare Nähe gerückt war. Die Naivität eines rein linear-quantitativ orientierten Bewußtseins wurde bewußt, aber auch, daß wirtschaftliches Denken einen ethischen Ausgangspunkt hat: das Suchen nach Wohlstand und Wohlbefinden für Menschen.

Wissenschaft und Technik produzieren Güter und vertreiben sie, ohne nach ihrer Vernünftigkeit zu fragen. Sie beschränken sich auf „instrumentelle Vernunft"-Methoden, die einer Maximierung des wirtschaftlichen Wachstums dienen. Vergessen wird, daß es jenseits dieses vordergründigen Zweckes verpflichtende Grundziele gibt: Beseitigung von menschenunwürdigen Arbeitsbedingungen, soziale Sicherheit, Wohlstand, Frieden.

Die anthropologischen und ethischen Zielvorstellungen, über die ein Grundkonsens erreicht werden muß, lauten:

a. Ziel aller wissenschaftlichen, technischen und wirtschaftlichen Bemühungen ist die Selbstverwirklichung der Menschen, also die Entfaltung als Personalität in Sozialität und Materialität. Der Mensch muß sich in allen drei Dimensionen seines Daseins verwirklichen können. Jede Technik und Wissenschaft ist daran zu messen, inwieweit sie hilft, daß sich die Menschen im Rahmen ihrer sozialen und materiellen Bedingungen entfalten können. Der Mensch ist Maßstab der Technik, nicht aber die Technik Maßstab des Menschen.

b. Die Richtung menschlicher Selbstverwirklichung ist in jüngster Gegenwart mit groBer Deutlichkeit bewußt geworden: der Mensch ist nach vorn, nach vorwärts verwiesen. Er ist nicht nur legitimiert, Krankheiten und Schwächen zu therapieren. Das Ereignis der Evolution selbst ist verantwortlich in die Hand des Menschen gelegt. Als „Vorhut und Gestalter“35 der Evolution wird der Mensch aber nur gerecht, wenn er allen anderen Gütern und Werten die Werte der Freiheit, der Liebe, der Gerechtigkeit, der Gleichheit und der Solidarität vorausstellt.

c. In jedem Einzelfall ist über Mittel und Wege zum Ziel menschlicher Selbstverwirklichung nach dem Prinzip der Güterabwägung zu entscheiden. Es ist sowohl auf die Werthöhe als auch auf die Wertdringlichkeit von Sinnzielen und konkreten Zwecken zu achten. Die Ausarbeitung von Kriterien für einzelne Bereiche menschlicher Daseinsgestaltung (wie der Medienethik) ist eine wichtige, aber noch ungelöste Aufgabe der Ethik.

d. Wissenschaft und Technik müssen wieder eingebunden werden in religiös und ethisch begründete Sinngebung. Theologische Überlegungen sind nicht unwissenschaftlich, weil nicht naturwissenschaftlich. Jede Naturwissenschaft darf sich nicht bloß auf ihre empirischen Methoden verlassen, sondern muß den Sinn und das Ziel ihres Tuns mitverantworten.

e. Wenn das Gekonnte nicht auch schon das Gesollte ist, wenn die technische Machbarkeit einer Sache nicht bereits die Legitimation ihrer Realisierung ist, dann gilt als übergeordnete Bedingung, daß „homo homini sanctus“ ist:

- wenn die Selbstwertigkeit des Einzelnen nicht tangiert wird, indem er manipuliert oder als manipulierbares Material behandelt wird oder wenn er als Kostenfaktor oder austauschbare Sache angesehen wird;

- wenn die Fähigkeit, sich selbst zu bestimmen, stabile soziale Beziehungen aufzu- 
bauen und sich an der Gestaltung der öffentlichen Angelegenheiten zu beteiligen, ungeschmälert möglich ist;

- wenn die Realisierung des Machbaren nicht unkontrollierte Beeinflussungsmöglichkeiten und Abhängigkeiten schafft;

- wenn die Kehrseite des Fortschritts (absehbare Neben- und zu befürchtende Spätfolgen) nicht die Lebensmöglichkeiten unserer Kinder und Kindeskinder irreversibel beeinträchtigen. ${ }^{36}$

f. Gegenüber der Verantwortbarkeit einer Sache muß der Umstand, daß aus ihr ökonomischer Gewinn gezogen werden kann, zweitrangig bleiben.

\section{Schlußwort}

In einem Vortrag aus Anlaß des Papstbesuches 1980 hat Professor Dr. Carl Friedrich von Weizsäcker ein Zitat aus der Rede des Papstes vor der Unesco hervorgehoben, ein Zitat nach Thomas von Aquin, das eine Erkenntnis von Aristoteles aktualisiert: „Genus humanum arte et ratione vivit ${ }^{437}$. Das Menschengeschlecht lebt durch Kunstfertigkeit und Vernunft - sofern nämlich beide vereinbar sind. So zu leben macht Kultur aus, weil nicht das, was der Mensch hat, sondern was der Mensch ist, seine Menschheit ausmacht und Kultur das ist (oder sein soll!), was den Menschen als solchen mehr Mensch sein läßt. Weizsäcker zitiert Johannes Paul II. wörtlich: „Es besteht kein Zweifel, daß das erste und grundlegende kulturelle Faktum der geistig reife Mensch ist, das heißt der vollerzogene Mensch, der Mensch, der fähig ist, sich selbst und andere zu erziehen. Es besteht kein Zweifel, daß die erste und grundlegende Dimension der Kultur ihre gesunde Moral ist: also die moralische Kultur". Diesem moralischen Appell des Papstes fügte Weizsäcker zwei Forderungen an: 1. Es muß politische Entscheidungsträger geben, die das Notwendige beschließen und durchsetzen können. 2. Diese Entscheidungsträger müssen die notwendige Einsicht und den notwendigen Willen haben.

Es steht außer Zweifel, daß uns die sogenannten Neuen Medien, besser die Informations- und Kommunikationstechniken, in ihren Anwendungen und Auswirkungen beeinflussen werden. Das hat Auswirkungen auf unser bisheriges Selbstverständnis in einer Form, die uns kaum bewußt ist. Darüber nachzudenken wird es höchste Zeit. Welche Ziele wollen wir eigentlich wie und warum in der Informationsgesellschaft verfolgen? Es ist dann wirklich zu fragen: wie verstehen wir uns und wozu leben wir, um im einzelnen entscheiden zu können; was sollen wir warum wollen, wenn wir es technisch können?

Der Weg in eine sinnvolle Informationsgesellschaft führt verantwortbar nur über die Anthropologie und die Ethik. Ziel, Wege und Methoden humanen Seins bedürfen wieder einer Gesamtschau, wissenschaftsbereichübergreifend und das antiquierte naturwissenschaftlich-empirische Denken aufsprengend. Das Bedenken dieser Fragen ist überfällig, die Diskussion dringlich. Wenn Christen aus dem Prinzip Hoffnung heraus zu leben behaupten, dann haben doch gerade sie Anlaß und Verpflichtung, über tragfähige und sinnvolle Konzeption für Sozietäten und Individuen, Staat und Familie nachzudenken. Wie aber eine - vielleicht auch nur partiell - christliche Gesellschaft morgen aussehen soll, wird heute konzipiert! 
0 B. Brecht: Geschichten von Herm Keuner. Frankfurt 1985, 33

l Vgl. den Aufsatz von 1979: Die Antiquiertheit der philosophischen Anthropologie. In: G. Anders: Die Antiquiertheit des Menschen. München ${ }^{3} 1984$, Bd. 2, $128 \mathrm{ff}$.

2 Der Stil, in dem Anders ebd. die Wesensfrage als Vorurteil und unbeantwortete Selbstgefälligkeit behandelt, entlarvt die Empfindsamkeit des Autors gegenüber dem Umstand, daß sich ein Mensch nicht ohne negative Folgen auf seine bloße Rationalität relativieren läßt.

3 Ebenfalls bei Anders ist schon die „Hochnäsigkeit“ (ebd., 129) kritisiert, eine Sonderstellung des Menschen im Kosmos anzunehmen. Von hier bis zu den in aller Deutlichkeit bei H. v. Ditfurth erhobenen Forderungen, der Mensch könne sich angesichts der von ihm zu schaffenden evolutionären Fortentwicklung aus der Geschichte entfernen, ist es ein nur kurzer aber konsequenter Schritt.

4 Eine kleine, ungeordnete Auswahl von Termini, in denen das jeweilige anthropologische Verständnis kulminiert:

homo sapiens

homo faber

homo hominans

homo hominatus

homo inveniens

homo ludens

homo televisioniensis

homo informatus

homme machine (frz.)

animal creativum

animal educandum

animal habens

animal metaphysicum

animal symbolicum

animal indigentia

das nicht festgestellte Tier

eine offene Frage

ein Mängelwesen

ein nackter Affe der weise Mensch (Gattungsbegriff)

der herstellende Mensch (Bergson)

der sich hervorbringende Mensch

der durch sich hervorgebrachte Mensch

der erfinderische Mensch

der spielende (=spielfähige) Mensch

der fernsehende Mensch

der informierte Mensch

der Mensch als Maschine (= Maschinenmensch)

das schöpferische Wesen

das Wesen, das erzogen werden muß

das Wesen, das "hat“ (Anders)

das metaphysische Wesen

das symbolische (= symbolfähige) Wesen

das bedürftige Wesen

(Nietzsche)

Plessner

Gehlen

Neben diesen Termini, in denen verschiedene Auffassungen vom Menschen wie im Brennglas zusammengefaßt sind und neben den gelehrten anthropologischen Abhandlungen existieren natürlich noch weitere, zahlreiche Möglichkeiten, ein Bild vom Menschen zu formen.

5 Der Umstand, daß es nicht „die Anthropologie“ gibt, an der unterschiedliche Anthropologen im Lauf der Geschichte mitwirken, läßt sich auch wie folgt erklären: Die Anthropologien sind ein Produkt des Menschen und die Ansichten, die sich aus ihnen ergeben, sind vermittelt - sei es durch den Apparat des Gehirns, durch soziale Strukturen oder historische Bedingungen. Umgekehrt läßt sich auch so feststellen, daß die Anthropologien selbst ein Produkt des veränderten Verständnisses des Menschen von sich selbst sind.

$6 \mathrm{Vgl.:}$ Hartenstein (Hrsg.): Kant's sämtliche Werke. Leipzig: Leopold Voss 1968, Bd. VIII, 24 f.

7 R. Lassahn: Pädagogische Anthropologie. Eine historische Einführung. Heidelberg 1983. Hier 9. Synonym verwendet Lassahn für „implizite Anthropologie“ auch „Kryptoanthropologie“.

8 Ebd., 144

9 Obwohl die Leistung eines Autos heute nach KWh angegeben wird, messen die meisten Menschen noch nach Pferdestärken; die Formel zur Leistungsberechnung stammt von Watt: ein Pferd zieht $75 \mathrm{~kg}$ in einer Sekunde 1 Meter hoch.

10 Lassahn, 156 
11 „Homologisierung“ ist eine Begriffsbildung von R. Lassahn (Pädagog. Anthropologie, S. 149 f.). Abgeleitet von "homolog"(Duden: gleichliegend, gleichlautend, übereinstimmend, entsprechend) bezeichnet er damit die Übertragung naturwissenschaftlicher, empirischer Termini und Modelle auf den Menschen. Der von der Elektrizitätslehre exakt definierte Begriff "Spannung", meßbar und nach naturwissenschaftlichen Gesetzen ablaufend, wird in andere Lebensbereiche übertragen. Die homologe Verwendung von „Spannung“ (z.B. Spannung zwischen zwei Menschen) hat zur Folge, daß nicht nur der Begriff, sondern auch seine näheren Bedingungen homologisiert werden: das herangezogene Beispiel intendiert die Annahme, auch die „Spannung“ $z$ wischen zwei Menschen sei exakt meßbar und laufe gesetzmäßig ab. Homologisierung meint somit die Verwendung eines naturwissenschaftlichen Begriffs im zwischenmenschlichen Bereich unter der Annahme, eine geistige Tätigkeit nach einem analogen naturwissenschaftlichen Modell aufbauen zu können.

12 R. Lassahn, 156

13 Norbert Wiener (1894-1964), einer der geistigen Väter des Computers, begründete die Kybernetik. Vgl. z.B.: Mensch und Menschmaschine. Berlin 1958

14 R. Lassahn, 156

15 Ebd. 157

16 K. Steinbuch: Falsch programmiert. Stuttgart 1969, 103

17 R. Lassahn, 157

18 G. Günther. In: Grundlagenstudium aus Kybernetik und Geisteswissenschaften, Bd. 1, 1960, 41 (nach R. Lassahn, 158)

19 Vgl. A. Bammé, G. Feuerstein u.a. (Hrsg.): Maschinen-Menschen, Mensch-Maschinen. Grundrisse einer sozialen Beziehung. Reinbek 1983

20 die erschreckenden medizinischen Horrorparallelen, die Konstruktion des schönen neuen Menschen infolge der Genmanipulation seien hier nur erwähnt, nicht vertieft.

21 Indiz für diese Behauptung ist der Umstand, in welchem A usmaß es zutrifft, daß „Information“ bereits zur Ware wurde, wie folgenschwer der Arbeitsmarkt sich neu zu strukturieren beginnt und die Ahnung von G. Anders, der Mensch werde den Menschen als Arbeiter überflüssig machen.

22 Eine Auswahl der inzwischen unüberschaubaren Publikationen zum Thema bietet: $M$. BeckerHuberti (Hrsg.): Neue Medien, Informations- und Kommunikationstechniken. Eine AuswahlBibliographie. Düsseldorf 1985. (zu erhalten über: Kath. Btx-Redaktion Düsseldorf, Hubertusstr. 5, 4000 Düsseldorf 1, Tel. 0211-307091).

23 Von einem Schweizer Informatiker stammt der folgende Vergleich: „Übertrüge man diesen Fortschritt (gemeint ist der Fortschritt der Informationstechnologie) auf den Automobilbau, so dürfte heute ein Mittelklassewagen noch DM 10.- kosten, er könnte 10.000 km in der Stunde zurücklegen, 5.000 Personen befördern und $1 / 2$ Liter Benzin müßte für $1.000 \mathrm{~km}$ reichen". Nach Klaus Brepohl: Telematik. Die Grundlage der Zukunft. Bergisch-Gladbach 1982, 49.

$24 \mathrm{~J}$. Weizenbaum: Computer: das neue Werkzeug. In; Zeitungskolleg Mikroprozessoren, $11 \mathrm{f}$.

25 Man denke nur daran, daß der weitaus größte Teil aller Verträge der menschlichen Unterschrift bedarf, die sich - vor allem nicht original - über Kabel übertragen läßt. (Was ist überhaupt noch Original, was Kopie?) Ist ein elektronischer Brief, der eine öffentliche Behörde außerhalb der Dienstzeiten erreicht, noch revidierbar?

26 Nach W.Steinmüller: Informationstechnologien und gesellschaftliche Macht. In: Zeitungskolleg Mikroprozessoren, $180 \mathrm{ff}$.

$27 \mathrm{Vgl}$ : $G$. Anders: Die Welt als Phantom und Matritze. In: Die Antiquiertheit des Menschen. München 1983, Bd. 1, 97.

28 Diese Kurse werden hochprozentig und fast ausschließlich von männlichen Schülern frequentiert, was zu gesellschaftsspezifischen Ungleichheiten führen wird.

29 Instrumentelles Denken meint das empirische Denken von Mathematik und Naturwissenschaft: in Kettenschlüssen und mit logischen Ableitungen. Topisches Denken meint das nichtsequentielle Denken, das Einbeziehen persönlichen Erlebens, der Intuition, Kreativität, Phantasie. Weiter meint es die einem Algorithmus unzugängliche Welt von logischer Unschärfe, unlogischer Verknüpfung, die Wohltat des Vergessenkönnens. 
30 Die Sprachcomputer eines deutschen Verlages, der sich mit Fremdsprachen befaßt, wenden sich der Sprache zu, die einen großen Absatz der Geräte erlauben. Die Methode der Gerätefunktion wurde vom Verlag festgelegt, ebenso die Auswahl und Zahl der verwendeten Worte aus der Allgemeinsprache. Die preiswerte Verfügbarkeit eines solchen Systems macht Konstruktion und Vertrieb von Alternativen unwahrscheinlich.

31 Das Naturbild der heutigen Physik. In: Die Künste im technischen Zeitalter. Hrsg. von der Bayer. Akademie der schönen Künste. Darmstadt 1952, 67.

32 K. Jaspers: Die Atombombe und die Zukunft des Menschen. München 1958

33 A. Auer: Darf der Mensch, was er kann? In: Wissenschaft-Technik-Humanität. Beiträge zu einer konkreten Ethik. Hrsg. von A.J. Buch und J. Splett. Frankfurt 1982, 11-35, hier 15 f.

34 Ebd., 16

35 T Dobzhansky, zitiert bei B. Häring: Ethik der Manipulation. Graz-Wien-Köln 1977, 86.

36 Vgl. K. Hilpert: Darf der Mensch, was er kann? In: M. Becker-Huberti (Hrsg.): Chips und Chancen. Entwicklungen, Daten und Gefahren. Eine Zeitungsserie der Neuß-Grevenbroicher Zeitung vom 23. Februar bis 27. April 1985. Neuss 1985, 38.

37 F. v. Weizsäcker: Glaube und wissenschaftlich-technische Welt. Vortrag aus Anlaß des Papstbesuches 1980. (Sonderdruck) Köln 1980. 


\section{SUMMARY}

The author discusses the inter-relations between the philosophical/anthropological question of what is man, and the new media. First it is shown that there exists an implicit anthropology, a socalled "crypto anthropology" which has adapted scientific terms and concepts; since long, the assumptions of natural sciences (e.g. linear thinking, reduction on empirical reality, „yes" or "no“ thinking, etc), frame the way of human self reflection. Deriving however his self understanding from the function of machines, man reduces himself substantially. The author therefore pleads for re-thinking anthropolgy and ethics, and mainly appeals to the ability of man very early to learn about functioning, facilities and non-facilities of application, organisation, and structures of media and information technology. Not the media and technology must define man, he himself has to define in order to use these media appropriately.

\section{RÉSUMÉ}

Lauteur discute ici le rapport entre la question philosophico-anthropologique de l'homme et les nouveaux médias. Tout d'abord, il montre l'existence d'une anthropologie implicite, une „cryptoanthropologie“, qui adapte des concepts et modèles des sciences physiques et naturelles. Depuis longtemps, les présuppositions des sciences naturelles (pensée linéaire et dichotomique, empirisme, etc.) constituent un des cadres de références de la compréhension de l'homme par lui-même. Pourtant, en appliquant le fonctionement des machines à sa propre compréhension, l'homme réduit sa propre essence. C'est pourquoi, l'auteur plaide en faveur d'une anthropologie et d'une éthique nouvelles, pour un apprentissage précoce par l'homme des fonctions et des possibilités d'utilisation, de l'organisation et des structures des médias et des techniques d'information et de communication. Non que les médias et les technologies doivent définir l'homme, au contraire, c'est à l'homme de se connaître pour pouvoir utiliser ces médias de la façon la plus appropriée.

\section{RESUMEN}

En este artículo el autor se refiere a la relación entre la cuesstión filosófica-antropológica del Hombre y los nuevos media. Primeramente él demuestra la existencia de una antropología implicita, una „cripto-antropología“ que adapta los conceptos y modelos de las ciencias fisicas y naturales. Desde hace mucho tiempo las presuposiciones de las ciencias naturales (pensamiento lineal y dicotómico, empirista, etc. .) constituyen uno de los cuadros de referencia de la comprensión del hombre por si mismo. Sin embargo, aplicando el principio del funcionamiento de las máquinas a su propia comprensión, el hombre ha reducido su propie esencia. Por esta razón el autor propone y apoya una nueva antropología y ética, un aprendizaje precoz del hombre de las funciones y de las posibilidades de utilización, de la organización y de las estructuras de los media y de las técnicas de información y comunicación; no porque los media deban definir al hombre, al contrario, el hombre debe conocerse bien a si mismo para utilizar estos de la manera más apropiada. 